\title{
Comparative calorimetric study of non-amyloidogenic and amyloidogenic variants of the homotetrameric protein transthyretin
}

\author{
Valery L. Shnyrov, ${ }^{\mathrm{a}, 1, *}$, Enrique Villar ${ }^{\mathrm{a}}$, Galina G. Zhadan ${ }^{\mathrm{a}}$, \\ Jose M. Sanchez-Ruiz ${ }^{\mathrm{b}}$, Alexandre Quintas ${ }^{\mathrm{c}, \mathrm{d}}$, Maria João M. Saraiva ${ }^{\mathrm{e}}$, \\ Rui M.M. Brito ${ }^{\mathrm{c}, \mathrm{f}}$ \\ ${ }^{a}$ Departamento de Bioquímica y Biologia Molecular, Universidad de Salamanca, 37007 Salamanca, Spain \\ ${ }^{\mathrm{b}}$ Facultad de Ciencias, Departamento de Química-Física e Instituto de Biotecnologia, 18071 Granada, Spain \\ ${ }^{\mathrm{c}}$ Centro de Neurociencias de Coimbra, Universidade de Coimbra, 3000 Coimbra, Portugal \\ ${ }^{\mathrm{d}}$ Instituto Superior de Ciencias da Saude Sul, Quinta da Granja, 2825 Monte da Caparica, Portugal \\ ${ }^{\mathrm{e}}$ Instituto de Ciencias Biomedicas de Abel Salazar and Amyloid Unit, Institute for Molecular and Cellular Biology, \\ Universidade do Porto, 4050 Porto, Portugal \\ ${ }^{\mathrm{f}}$ Departamento de Quimica, Faculdade de Ciencias e Tecnologia, Universidade de Coimbra, 3049 Coimbra, Portugal
}

Received 30 May 2000; received in revised form 3 August 2000; accepted 4 August 2000

\begin{abstract}
Familial amyloidotic polyneuropathy (FAP) is an autosomal dominant hereditary type of amyloidosis involving amino acid substitutions in transthyretin (TTR). V30M-TTR is the most frequent variant, and L55P-TTR is the variant associated with the most aggressive form of FAP. The thermal stability of the wild-type, V30M-TTR, L55P-TTR and a non-amyloidogenic variant, T119M-TTR, was studied by high-sensitivity differential scanning calorimetry (DSC). The thermal unfolding of TTR is a spontaneous reversible process involving a highly co-operative transition between folded tetramers and unfolded monomers. All variants of transthyretin are very stable to the thermal unfolding that occurs at very high temperatures, most probably because of their oligomeric structure. The data presented in this work indicated that for the homotetrameric form of the wild-type TTR and its variants, the order of stability is as follows: wild-type TTR $z$ T119M-TTR $>$ L55P-TTR $>$ V30M-TTR, which does not correlate with their known amyloidogenic potential. (C) 2000 Elsevier Science B.V. All rights reserved.
\end{abstract}

Keywords: Transthyretin; Familial amyloidotic polyneuropathy; Differential scanning calorimetry; Thermal stability

\footnotetext{
* Corresponding author. Tel.: +34-923-294465; fax: +34-923-294579.

E-mail address: shnyrov@gugu.usal.es (V.L. Shnyrov).

${ }^{1}$ Permanent address: Institute of Theoretical and Experimental Biophysics, Russian Academy of Sciences, 142292 Pushchino, Moscow region, Russia.
} 


\section{Introduction}

Human transthyretin is a homotetrameric protein with a total molecular weight of $55 \mathrm{kDa}$ and 127 amino acid residues per subunit [1] that is found in cerebrospinal fluid and in plasma [2]. TTR transports thyroxine and retinol in association with a retinol-binding protein [3-7].

Recent interest in TTR was brought about by the discovery that single-site mutants of TTR are the major protein component of amyloid fibrils in FAP, a lethal autosomal dominant disease [8]. TTR with a valine replaced by methionine at position 30 (V30M-TTR) produces the most frequent form of FAP $[9,10]$, and TTR with leucine at position 55 replaced by a proline (L55P-TTR) is the variant associated with the most aggressive form of amyloidosis [11]. Transthyretin with threonine at position 119 replaced by a methionine (T119M-TTR) was originally described in a kindred form without amyloidosis [12] and has now been found in several human populations [13]. This variant is non-amyloidogenic and is thought to protect individuals who also carry the V30M mutation against FAP [14].

A comparison between the crystal structures of the wild-type TTR (WT-TTR) and the V30M-TTR variant revealed a very similar global fold for both proteins, the tetramer having a central cylindrical cavity in which thyroxine binding occurs and an exterior binding site for the retinol-binding protein. Each subunit consists of two $\beta$-sheets with four $\beta$-strands each, forming a $\beta$-sandwich $[15,16]$. Substitution of valine 30 by methionine forces the $\beta$-sheets of the monomer approximately $1 \AA$ apart, resulting in a distortion of the thyroxine binding cavity $[15,16]$. However, the small differences between the crystal structures of WT-TTR and V30M-TTR cannot be satisfactorily invoked to account for the amyloidogenicity of V30M-TTR. In contrast, the recently solved crystal structure of L55P-TTR points to significant structural differences from WT-TTR [17]. In particular, the small D strand does not exist in L55P-TTR.

Differential scanning calorimetry, which is an extremely useful technique for characterizing the energetics of the thermal unfolding of proteins [18-20], was used here to evaluate potential con- formational stability differences between the above-mentioned amyloidogenic and nonamyloidogenic transthyretin variants, WT-TTR, V30M-TTR, L55P-TTR and T119M-TTR. The results obtained demonstrated that all the TTR variants had very high transition temperatures $\left(T_{m}\right)$ for thermal unfolding and all of them unfolded and dissociated simultaneously, obeying a two-state mechanism between the folded tetrameric state and the unfolded monomeric state, according to the scheme $\mathrm{N}_{4} \rightleftarrows 4 \mathrm{U}$.

\section{Experimental}

\subsection{Materials}

Recombinant TTR variants were produced in an Escherichia coli expression system [21] and purified as described previously [22]. Protein concentrations were determined spectrophotometrically at $280 \mathrm{~nm}$, using an extinction coefficient of $7.76 \times 10^{4} \mathrm{M}^{-1} \mathrm{~cm}^{-1}$ based on a $55-\mathrm{kDa}$ molecular weight for the TTR tetramer [6]. TTR variants in the tetrameric form were prepared by gel exclusion chromatography, as described previously [23].

All chemicals were of analytical grade, with the highest purity commercially available and were purchased from Sigma (St. Louis, MO). Doubledistilled water was used throughout.

\subsection{Differential scanning calorimetry}

DSC experiments were performed on a MicroCal MC-2D differential scanning microcalorimeter (MicroCal Inc., Northampton, MA) with cell volumes of $1.22 \mathrm{ml}$, as described previously by Ruiz-Arribas et al. [24]. Sample and reference solutions were properly degassed with stirring in an evacuated chamber for $5 \mathrm{~min}$ at room temperature and then carefully loaded into the calorimeter cells. An overpressure of $2 \mathrm{~atm}$ of dry nitrogen was always kept over the liquids in the cells throughout the scans to prevent any degassing during heating. Exhaustive cleaning of the cells was undertaken before each experiment. A background scan collected with buffer in both cells 
was subtracted from each scan. The reversibility of the thermal transitions was verified by checking the reproducibility of the calorimetric trace in a second heating of the samples immediately after cooling from the first scan. The temperature dependence of the molar heat capacity of the proteins was further analyzed by a non-linear least-squares fitting and plotted using the Windows-based software package (Origin) supplied by MicroCal.

The heat capacity of TTR and its changes were parameterized in terms of accessible surface area (ASA) [25] in accordance with Eqs. (1) and (2) [26]:

$C_{p}=C_{p, a}+C_{p, b}+C_{p, c}$

$$
\begin{aligned}
\Delta C_{p}=\Delta C_{p, a p}+\Delta C_{p, p o l}= & a_{c}(t) \cdot \Delta A S A_{a p} \\
& +b_{c}(t) \cdot \Delta A S A_{p o l}
\end{aligned}
$$

Each term in Eq. (1) is given by:

$C_{p, a}=[0.28+0.000975 \cdot(t-25)] \cdot M W$

$C_{p, b}=[0.0087+0.000643 \cdot(t-25)] \cdot B S A_{\mathrm{tot}}$

$C_{p, c}=\left[a_{c}(T) \cdot A S A_{a p}+b_{c}(T)\right] \cdot A S A_{\mathrm{pol}}$

where the coefficients

$$
\begin{aligned}
a_{c}(t)= & 0.45+0.000263 \cdot(t-25) \\
& -0.000042 \cdot(t-25)^{2} \\
b_{c}(t)= & -0.26+0.000285 \cdot(t-25) \\
& +0.0000431 \cdot(t-25)^{2}
\end{aligned}
$$

In the above equations, MW is the molecular weight; $\mathrm{BSA}_{\text {tot }}$ is the total area buried from the solvent; ASA (BSA, $\triangle$ ASA) is in $\AA^{2}$, heat capacity is in cal $\mathrm{K}^{-1} \mathrm{~mol}^{-1}$, and $t$ is in ${ }^{\circ} \mathrm{C}$.

The ASA of the native and denatured states of WT-TTR and V30M-TTR, for which crystallographic coordinates with a resolution of $1.7 \AA$ were available [16], was calculated using MOLE software (Applied Thermodynamics LLC, Hunt Valley, MD). A 1.4- $\AA$ probe radius was employed. The ASA of the denatured state was taken as the sum of the areas of each residue in an extended Gly- $X$-Gly tripeptides, in which the side-chain of the central residue was in the same conformation as in the native protein $[25,26]$.

\section{Results and discussion}

Fig. 1 shows the partial molar heat capacity function of WT-TTR at a concentration of $25 \mu \mathrm{M}$ (here, TTR concentrations are always expressed in terms of monomer units) in $20 \mathrm{mM}$ sodium phosphate, $\mathrm{pH} 7.0$, in the presence of $150 \mathrm{mM}$ $\mathrm{NaCl}$. Under the conditions of this experiment, the thermal denaturation transition of WT-TTR occurred at $101.7^{\circ} \mathrm{C}$ and was characterized by an enthalpy change $\left[\Delta H\left(T_{m}\right)\right]$ of $96.8 \mathrm{kcal}$ (mol of monomer) ${ }^{-1}$ and a change in the heat capacity of $1.22 \pm 0.02 \mathrm{kcal} \mathrm{K}^{-1} \mathrm{~mol}^{-1}$. Fig. 1 also shows that the heat capacities of both the native and unfolded WT-TTR were in a good agreement with the values calculated using a structure-based parameterization (see Section 2). The similarity of the heat capacity of the denatured TTR and the heat capacity expected for an unstructured polypeptide of the same sequence suggested that the polypeptide chain was essentially unfolded and hydrated after thermal denaturation.

For a reliable reversible thermodynamic description of protein stability, it is necessary that the experimentally measured unfolding transition be reversible. The extent of reversibility, measured by the relative area recovery, seen on the second scan of TTR depended on the temperature at which the first scan was terminated before cooling the samples in preparation for the second scan. If the first scan allowed us to proceed up to a temperature at which the transition was $90 \%$ complete, then the repeated scan retained approximately $95 \%$ of the transitional enthalpy of the preceding one, indicating that the calorimetric experiments represented equilibrium conditions. However, prolonged incubation of the samples at high temperatures resulted in the appearance of an irreversible process. For example, after 


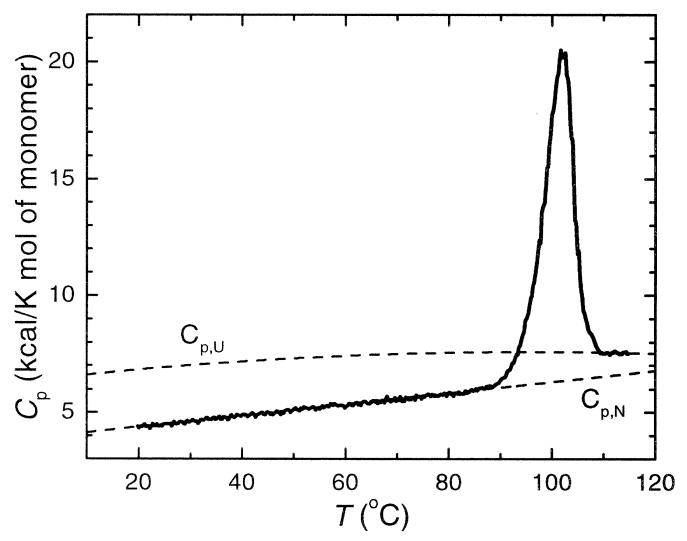

Fig. 1. Partial molar heat capacity of WT-TTR as function of temperature. The TTR concentration was $25 \mu \mathrm{M}$ of monomers. The scan rate was $1 \mathrm{~K} / \mathrm{min}$. The dashed lines represent the heat capacities of the native, $C_{p, N}$, and unfolded, $C_{p, U}$, states respectively, calculated as described in [26].

the incubation of $\mathrm{TTR}$ at $110^{\circ} \mathrm{C}$ or higher for longer than $5 \mathrm{~min}$ in repeated scans, only approximately $30 \%$ of the transitional enthalpy of the preceding scan was retained. Experiments performed at scanning rates of 0.5 and $1.5 \mathrm{~K} / \mathrm{min}$ gave denaturation profiles that differed by less than $0.2 \mathrm{~K}$, indicating the absence of kinetic effects under the conditions of our experiments.

At $25^{\circ} \mathrm{C}$, the experimentally determined heat capacity of the native state of TTR was $4.54 \pm 0.06$ $\mathrm{kcal} \mathrm{K}^{-1}$ (mol of monomer) ${ }^{-1}$ or $0.330 \pm 0.005$ cal $\mathrm{K}^{-1} \mathrm{~g}^{-1}$, which was similar to the mean value of $0.34 \pm 0.005 \mathrm{kcal} \mathrm{K}^{-1} \mathrm{~g}^{-1}$ obtained for globular proteins [26]. The enthalpy changes for the four studied variants of TTR at the same concentration $(25 \mu \mathrm{M})$ exhibited a temperature dependence consistent with the observed $\Delta C_{p}$ or $\Delta C_{p}$ calculated by Eq. (2) $\left(1.22 \pm 0.01 \mathrm{kcal} \mathrm{K}^{-1} \mathrm{~mol}^{-1}\right)$ and were equivalent $\left(1.23 \pm 0.03 \mathrm{kcal} \mathrm{K}^{-1} \mathrm{~mol}^{-1}\right)$ when evaluated at the same temperature (Fig. 2). Within the experimental temperature range of the experiments, the enthalpy change for the four TTR variants as a function of the temperature could be well represented by the equation:

$$
\Delta H(t)=94.44 \mathrm{kcal} \mathrm{mol}^{-1}+1.237
$$

$$
\mathrm{kcal} \mathrm{K}{ }^{-1} \mathrm{~mol} \cdot(t-100)
$$

with a regression coefficient of 0.999 and where $t$ is in ${ }^{\circ} \mathrm{C}$.

We also calculated the so-called generic enthalpy change, scaled in terms of $\triangle$ ASA changes at the reference temperature of $60^{\circ} \mathrm{C}$ in accordance with equation:

$$
\begin{aligned}
\Delta H_{\mathrm{gen}}(60)= & -8.44 \cdot \Delta \mathrm{ASA}_{a p} \\
& +31.4 \cdot \Delta A S A_{\mathrm{pol}}
\end{aligned}
$$

where the enthalpy change is in cal $\mathrm{mol}^{-1}$ [27].

Using the value of the heat capacity change, one can calculate enthalpy changes at a different temperature $\left(T\right.$ in $\left.{ }^{\circ} \mathrm{C}\right)$ as:

$$
\Delta H(T)=\Delta H(60)+\Delta C_{p} \cdot(T-60)
$$

Thus, the calculated value of $\Delta H$ for WT-TTR at $100^{\circ} \mathrm{C}$ is $160.8 \mathrm{kcal} \mathrm{mol}^{-1}$, almost two-fold higher than that obtained in the experiments. Currently, we have no explanation for this discrepancy.

Fig. 3 shows a set of DSC scans obtained for four different variants of TTR. The figure shows the excess heat capacity functions obtained after subtracting the heat capacity of the native state. It may be seen that all transition peaks were

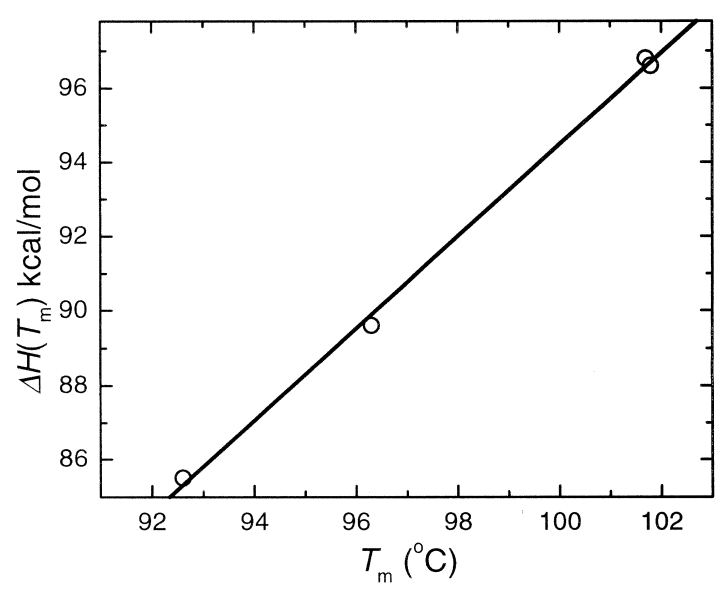

Fig. 2. The temperature-dependence of the enthalpy change for the four TTR variants at the same concentration of $25 \mu \mathrm{M}$ considered in this study. The four values define a straight line with a slope (heat capacity change) of $1.23 \mathrm{kcal} \mathrm{K}^{-1} \mathrm{~mol}^{-1}$; similar to the value obtained from the individual heat capacity curves. 


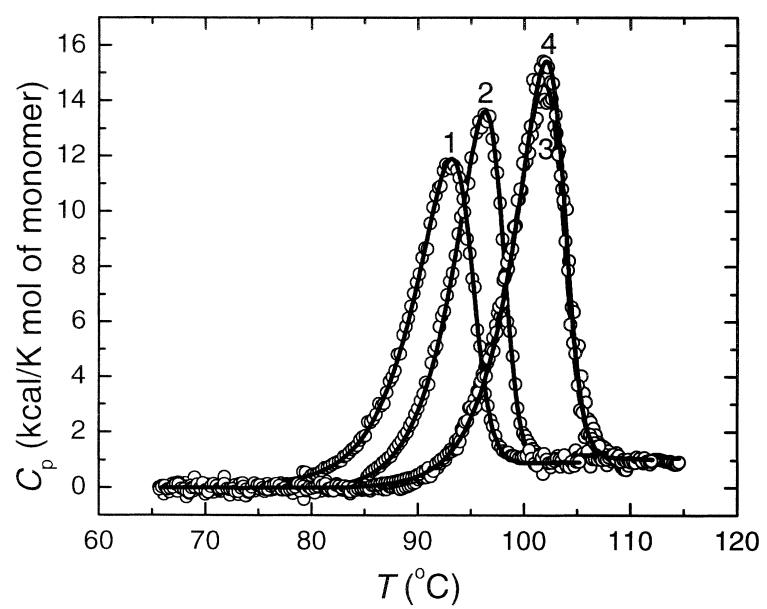

Fig. 3. Excess heat capacity of V30M-TTR (1), L55P-TTR (2), WT-TTR (3) and T119M-TTR (4) at pH 7.0 (symbols). The continuous lines represent the results of non-linear least square fittings of the excess molar heat capacity curves to the $\mathrm{N}_{4} \rightleftarrows 4 \mathrm{U}$ model as implemented in the Origin software package. Protein concentrations were $25 \mu \mathrm{M}$.

skewed toward the low temperature side of the transition, as expected for a transition coupled to dissociation according to the scheme $N_{m} \rightleftarrows m U$, where $m$ associated molecules of the native protein, $N$, give rise to $m$ molecules of the unfolded protein, $U$ [28]. Consequently, the temperature of the midpoint of the transition $T^{*}$ did not coincide with the temperature of the excess heat capacity maximum, $T_{m}$. For the calorimetric scans shown in Fig. 3, the ratio of the van't Hoff enthalpy [defined using the familiar formula $\Delta H_{V H}$ $\left.=4 R T_{m}^{2} C_{p}^{\max } / \Delta H\left(T_{m}\right)\right]$ and the calorimetric enthalpy $\left[\Delta H_{V H} / \Delta H\left(T_{m}\right)\right]$ averaged $1.66 \pm 0.08$. This value was significantly higher than 1.0 , which is the expected value for the two-state unfolding of a monomeric protein. For the two-state unfolding of an oligomeric protein coupled to a dissociation process [29]:

$\Delta H_{V H} / \Delta H\left(T_{m}\right)=2 m /(m+1)$

and equaled 1.6 for a tetramer, which was close to the obtained experimental value.

In a general coupling between the unfolding and dissociation processes, equilibrium thermodynamics predicted the following relationship between the total monomer concentration, $A_{t}$, and the unfolding temperature $T_{m}(\mathrm{~K})[29,30]$ :

$$
\begin{aligned}
\ln A_{t}= & \text { constant }-m \Delta H\left(T_{m}\right) \\
& \times(m-1)^{-1} R^{-1} T_{m}^{-1}
\end{aligned}
$$

where $\Delta H\left(T_{m}\right)$ stands for the unfolding enthalpy per monomer. The plots of $\ln A_{t}$ vs. $1 / T_{m}$ (Fig. 4) for tetramers of WT-TTR and V30M-TTR yielded straight lines. The values of $\Delta H\left(T_{m}\right)$ obtained from the slopes of these lines were $96 \pm 3 \mathrm{kcal}$ $\mathrm{mol}^{-1}$ for WT-TTR, and $83 \pm 3 \mathrm{kcal} \mathrm{mol}^{-1}$ for V30M-TTR, close to the values of calorimetric enthalpy, 96.8 and $85.5 \mathrm{kcal} \mathrm{mol}^{-1}$, respectively, for these proteins.

The software package (Origin) supplied by MicroCal afforded excellent fittings of our experimental data for $m=4$ (Fig. 3, continuous lines), whereas similar fittings for other $m$ values were considerably worse. The highest likelihood values for $T_{m}$ and $\Delta H\left(T_{m}\right)$ obtained by the fitting procedure are shown in Table 1.

All the above data allowed us to conclude that all the variants of TTR studied here behaved, in solution, as tetramers, which underwent a twostate reversible thermal unfolding coupled to dissociation, at least under our experimental conditions.

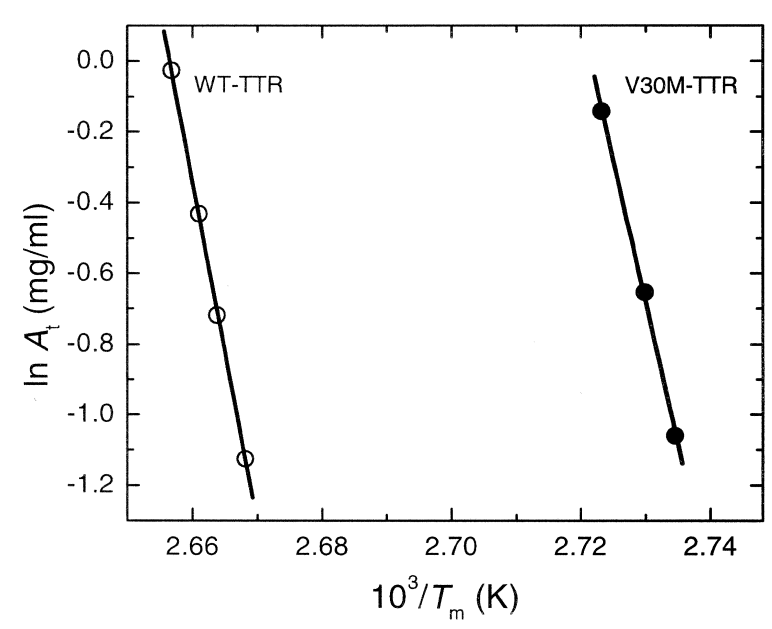

Fig. 4. Plots of the logarithm of the total protein concentration against $1 / T_{m}$, where $T_{m}$ is the absolute transition temperature for WT-TTR $(\bullet)$ and for V30M-TTR $(O)$. The lines were fitted by linear regression. 
Table 1

Comparison of the thermodynamic parameters for the unfolding of wild-type TTR and three of its variants ${ }^{\mathrm{a}}$

\begin{tabular}{|c|c|c|c|c|}
\hline Protein & $T_{m}\left({ }^{\circ} \mathrm{C}\right)$ & $T^{*}\left({ }^{\circ} \mathrm{C}\right)$ & $\begin{array}{l}\Delta H\left(T_{m}\right) \\
(\mathrm{kcal} / \mathrm{mol})\end{array}$ & $\begin{array}{l}\Delta \Delta G^{\mathrm{o}} \\
(\mathrm{kcal} / \mathrm{mol})\end{array}$ \\
\hline WT-TTR & $101.7 \pm 0.2$ & $100.9 \pm 0.1$ & $96.8 \pm 2.9$ & 0 \\
\hline V30M-TTR & $92.6 \pm 0.2$ & $91.9 \pm 0.2$ & $85.5 \pm 2.4$ & -2.24 \\
\hline L55P-TTR & $96.3 \pm 0.2$ & $95.1 \pm 0.1$ & $89.6 \pm 2.3$ & -1.47 \\
\hline T119M-TTR & $101.8 \pm 0.2$ & $100.7 \pm 0.2$ & $96.6 \pm 2.8$ & -0.05 \\
\hline
\end{tabular}

${ }^{\mathrm{a}} T_{m}$ is defined as the temperature at which the excess specific heat reaches its maximal value; $T^{*}$ is a temperature at which $50 \%$ of the protein is unfolded at a concentration of $1 \mathrm{mg} / \mathrm{ml} ; \Delta \Delta G^{\mathrm{o}}$ is the difference in the standard free energy change of unfolding, calculated as $\left(\Delta G_{\text {variant }}^{\mathrm{o}}-\Delta G_{\mathrm{WT}-\mathrm{TTR}}^{\mathrm{o}}\right)$ at $T^{*}$ for WT-TTR.

A convenient measure of the change produced by a mutation in the apparent stability of TTR is the quantity:

$\Delta \Delta G^{\mathrm{o}}=\left(\Delta G_{\mathrm{variant}}^{\mathrm{o}}-\Delta G_{\mathrm{WT}-\mathrm{TTR}}^{\mathrm{o}}\right)$

i.e. the difference in the standard free energies of unfolding for the variant and wild-type proteins, evaluated at $T^{*}$ [temperature at which $50 \%$ of protein is unfolded at exactly the same concentration $(25 \mu \mathrm{M})$ of protein] for the WT-TTR by means of the Gibbs-Helmholtz equation:

$$
\begin{aligned}
\Delta G^{\mathrm{o}}\left(T_{\mathrm{WT}-\mathrm{TTR}}^{*}\right)= & \Delta H_{\mathrm{WT} \text {-TTR }} \\
& \times\left(1-T_{\mathrm{WT} \text {-TTR }}^{*} / T_{\text {variant }}^{*}\right) \\
& -\Delta C_{p} \cdot\left[T_{\text {variant }}^{*}-T_{\mathrm{WT} \text {-TTR }}^{*}\right. \\
& +T_{\mathrm{WT} \text {-TTR }}^{*} \\
& \left.\times \ln \left(T_{\mathrm{WT} \text {-TTR }}^{*} / T_{\text {variant }}^{*}\right)\right]
\end{aligned}
$$

where $\Delta C_{p}$ is the denaturational heat capacity change, which we assumed to be constant since the temperature range of the extrapolation is small. It was obvious that in the present application of Eq. (11) that the term $\Delta \Delta G^{\mathrm{o}}\left(T_{\text {WT-TTR }}^{*}\right)=0$ and, hence, the destabilization of the native structure was indicated by a negative value for $\Delta \Delta G^{\circ}$ (Table 1).

The data shown in Table 1 indicated that for the wild-type TTR and its variants, the order of stability was as follows: WT-TTR $\succsim$ T119M-TTR $>$ L55P-TTR > V30M-TTR. This clearly showed that the tetrameric forms of non-amyloidogenic variants had a slightly higher conformational stability than amyloidogenic variants. However, the stability among the amyloidogenic variants did not correlate with the amyloidogenic potential of these proteins. Thus, the data obtained in this work were insufficient to explain amyloid fibril formation by TTR. It will, therefore, be necessary to examine more variants of TTR at the different levels of submolecular organization and under different experimental conditions.

\section{Acknowledgements}

This work was partly-supported by grant PRAXIS $/ 2 / 2.1 /$ SAU $/ 1287 / 95$, FCT, Portugal to R.M.M. Brito. A. Quintas is a doctoral fellowship holder from FCT, Portugal (GGPXXI/ BD/3237/96). We thank Mr Paul Moreira for technical assistance in preparing recombinant TTR.

\section{References}

[1] Y. Kanda, D.S. Goodman, R.E. Canfield, F.J. Morgan, J. Biol. Chem. 249 (1974) 6796-6805.

[2] C.C.F. Blake, I.D.A. Swan, C. Rerat, J. Berthou, A. Laurent, B. Rerat, J. Mol. Biol. 61 (1971) 217-224.

[3] J. Robbins, J.E. Rall, Physiol. Rev. 40 (1960) 415-489.

[4] M. Kanai, A. Raz, D.S. Goodman, J. Clin. Invest. 47 (1968) 2025-2049.

[5] A. Raz, T. Shiratori, D.S. Goodman, J. Biol. Chem. 245 (1970) 1903-1912.

[6] P.P. van Jaarsveld, H. Edelhoch, D.S. Goodman, J. Robbins, J. Biol. Chem. 548 (1973) 4698-4705.

[7] C. Yen, R.H. Costa, J.R. Darnell, J. Chen, T.A. Van Dyke, EMBO J. 9 (1990) 869-878.

[8] C. Andrade, Brain 75 (1952) 408-427. 
[9] F.E. Dwulet, M.D. Benson, Proc. Natl. Acad. Sci. USA 81 (1984) 694-698.

[10] M.J.M. Saraiva, J. Periph. Nerv. Syst. 1 (1996) 179-188.

[11] D.R. Jacobson, D.E. McFarlin, I. Kane, J.N. Buxbaum, Hum. Genet. 89 (1992) 353-356.

[12] H.H. Harrison, G.D. Gordon, W.C. Nichols, M.D. Benson, Am. J. Med. Genet. 39 (1991) 442-452.

[13] I.L. Alves, K. Atland, M.R. Almeida, P. Winter, M.J.M. Saraiva, Hum. Mutat. 9 (1997) 226-233.

[14] T. Coelho, R. Chorão, A. Sousa, I. Alves, M.F. Torres, M.J.M. Saraiva, Neuromusc. Disord. 6 (1996) 27-32.

[15] C.J. Terry, A.M. Damas, P. Oliveira et al., EMBO J. 12 (1993) 735-741.

[16] J.A. Hamilton, L.K. Steinrauf, B.C. Braden et al., J. Biol. Chem. 268 (1993) 2416-2424.

[17] M.P. Sebastião, M.J. Saraiva, A.M. Damas, J. Biol. Chem. 273 (1998) 24715-24722.

[18] P.L. Privalov, Adv. Protein Chem. 33 (1979) 167-241.

[19] A. Cooper, C.M. Johnson, in: C. Jones, B. Mulloy, A.H. Thomas (Eds.), Methods in Molecular Biology: Microscopy, Optical Spectroscopy, and Macroscopic Techniques, vol. 22, Humana Press, New Jersey, 1994, p. 109.
[20] V.L. Shnyrov, J.M. Sanchez-Ruiz, B.N. Boiko, G.G. Zhadan, E.A. Permyakov, Thermochim. Acta 302 (1997) 165-180.

[21] H. Furuya, M.J.M. Saraiva, M.A. Gawinowicz et al., Biochemistry 30 (1991) 2415-2421.

[22] M.R. Almeida, A.M. Damas, M.C. Lans, A. Brouwer, M.J. Saraiva, Endocrine 6 (1997) 309-315.

[23] A. Quintas, M.J.M. Saraiva, R.M.M. Brito, FEBS Lett. 418 (1997) 297-300.

[24] A. Ruiz-Arribas, G.G. Zhadan, V.P. Kutyshenko et al., Eur. J. Biochem. 253 (1998) 462-468.

[25] B. Lee, F.M. Richards, J. Mol. Biol. 55 (1971) 379-400.

[26] J. Gómez, V.J. Hilser, D. Xie, E. Freire, Proteins: Struct. Funct., Genet. 22 (1995) 404-412.

[27] V.J. Hilser, J. Gómez, E. Freire, Proteins 26 (1996) 123-133.

[28] S.P. Manly, K.S. Matthews, J.M. Sturtevant, Biochemistry 24 (1985) 384-3846.

[29] E. Freire, Comm. Mol. Cell. Biophys. 6 (1989) 123-140.

[30] K. Takahashi, J.M. Sturtevant, Biochemistry 20 (1981) 6185-6190. 